\title{
Research on the Water Temperature in the Bathtub Based on the Time-varying Model
}

\author{
Jiawen Li \\ North China Electric Power University, Baoding, 071000, China \\ china_almost@163.com
}

Keywords: the time-varying model, finite difference method, water temperature

\begin{abstract}
For the sake of providing scientific the theoretical basis for keeping the water temperature in the bathtub .we established the time-varying model of water temperature in the bathtub..By using finite difference method, the solution of the gained difference equations was given and simulated by computer. By changing parameters to make comparative analysis, we Further found the main factors that affect water temperature changes, and conducting optimal design too. Through calculation and analysis, we got the optimal bathtub size and put forward a series of Suggestions.
\end{abstract}

\section{Introduction}

In this section, we established the time-varying model of water temperature in the bathtub firstly; we explore the influence of bathtub (including superficial area, thickness, etc.) on water in bathtub. Then, we take bather into consider and explore the influence of bathing person (including action in the bathtub, human body, etc.) on water in bathtub. After that, we got what faucet flow velocity can make water temperature remain constant. Finally, we put forward the proposal to reduce the amount of water for bathing person.

\section{Natural Cooling Model of Bathtub Water}

We ignore the impact of bather into the water, and we can get some equations [1-2] as follow. Inverse radiative heat transfer of air and water on the surface of bathtub.

$$
\Phi_{b r}=\sigma \times \varepsilon_{w} \times\left(273.15+T_{w}\right)^{4} \times A
$$

Convective heat transfer between water and air

$$
\begin{gathered}
\Phi_{c}=0.47 f_{w z} \times\left(T_{w}-T_{a}\right) \times A \\
f_{w z}=9.2+0.46 W_{z}{ }^{2}
\end{gathered}
$$

Heat transfer of hot water and bath [2]

$$
\Phi_{a}=\left(T_{w}-T_{a}\right) \times S \times \frac{\lambda}{b}
$$

Then we can get the heat transfer of water and bath.

$$
C_{P 1} M \frac{d T_{w}}{d t}=-\sigma \times \varepsilon_{w} \times\left(273.15+T_{w}\right)^{4} \times A-0.47 f_{w z} \times\left(T_{w}-T_{a}\right) \times S \times \frac{\lambda}{b}
$$

\section{Simulating Results}

To meet people's need, tub baths should be of adequate size. We use standard bath size $2 \mathrm{~m} \times$ $0.8 \mathrm{~m} \times 0.5 \mathrm{~m}$. According to the actual situation; we can get the following data. Bring these data into the equation above, we can get Fig. 1.

As we can see from Fig. 1, the temperature of the water decreases over time, if you don't add water to the bathtub. After a period of time, water temperature reaches to the ambient temperature $T_{\mathrm{a}}$. We consider changing the numerical values of some parameters. Then, rate of temperature decreasing becomes slower. 


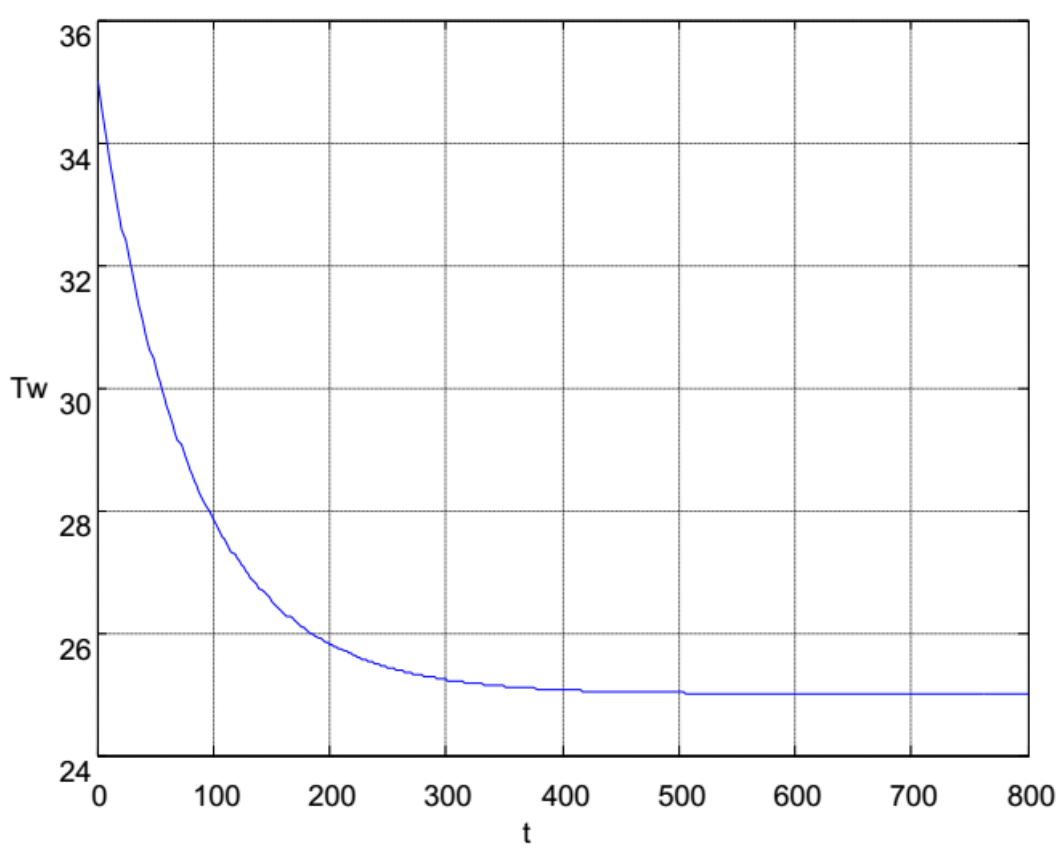

Fig. 1 Water temperature $T_{w}$ changes with time $t$

\section{Further Analysis of the Results}

Variable graph of changing parameter $b$ is shown as Fig.2. According to Fig.2. The changing rate of temperature becomes slower, with the increase of the bathtub's thickness . The time to reach the ambient temperature is prolonged. The conclusion is that the insulation effects depend on the thickness of bathtub. It provides a new way for us. We can increase the thickness of bathtub to achieve the purpose of keeping stable temperature. Moreover we can also reduce input of water as long as possible.

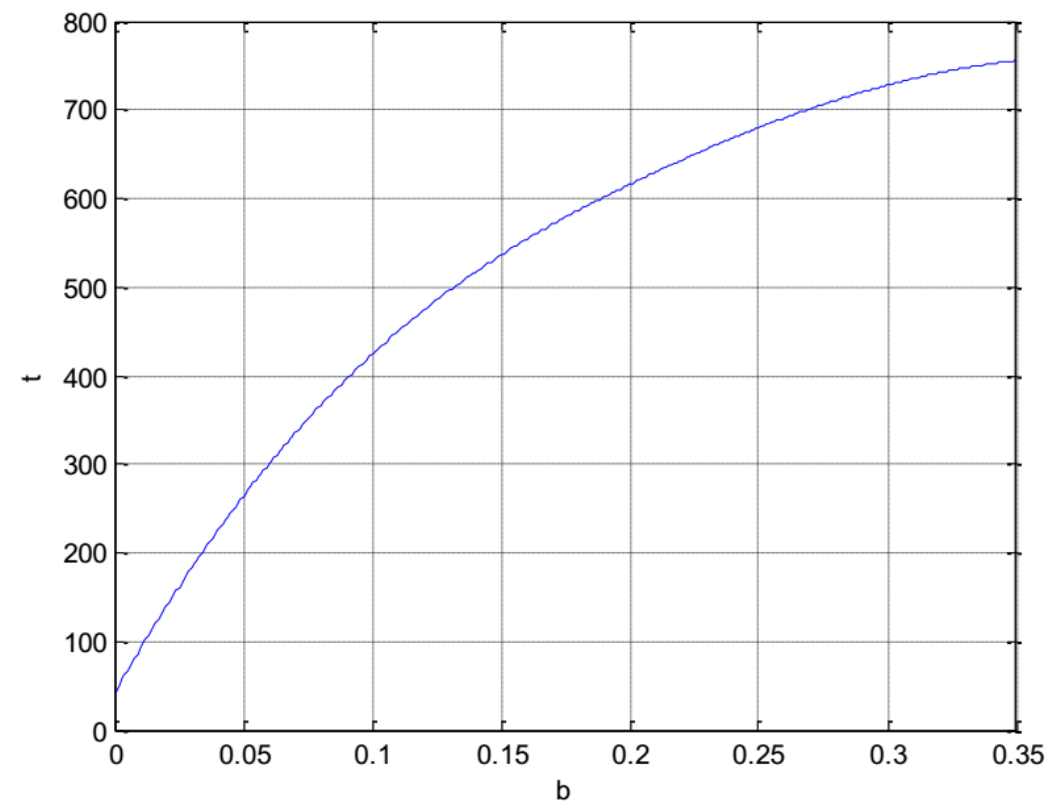

Fig. 2 Variable graph of changing parameter $b$

\section{The Model of Convection Transfer}

When the hot water feed into bathtub, the convective heat transfer between human and water can be represented as [3]:

$$
\Phi_{R}=\left(T_{w}-T_{R}\right) h_{c} S_{R}
$$

Method for Calculating Convection Heat Dissipation Coefficient. We can regard a person as 
a plane ellipse; shoulder width is to be the long axis of ellipse while the thickness of the person is to be short axis of ellipse. So we can get:

$$
h_{c}=B \sqrt{V}^{*}
$$

The relation between the temperature of the water in the bathtub and time changes to be as following.

$$
C_{P 1} M \frac{d T_{w}}{d t}=-\sigma \times \varepsilon_{w} \times\left(273.15+T_{w}\right)^{4} \times A-0.47 f_{w z} \times\left(T_{w}-T_{a}\right) \times S \times \frac{\lambda}{b}+W_{\eta}-\left(T_{w}-T_{R}\right) h_{c} S_{R}
$$

\section{Data Procession}

The body's movement of the bather will affect the metabolism rate and the consumption coefficient. As we all know, the more intense bather's movement is, the greater metabolism rate and the consumption coefficient are. Obviously, we can set some $W$ and $\eta$ to get the temperature's change over time.

Effect of the bather's movement on the temperature of the water is shown as Fig. 3. What we can see from the figure is that the rate of Temperature decrease get dropped, what's more, the time for the temperature's dropping become longer. So, we can conclude that People can increase the heat transfer to water with appropriate activities.

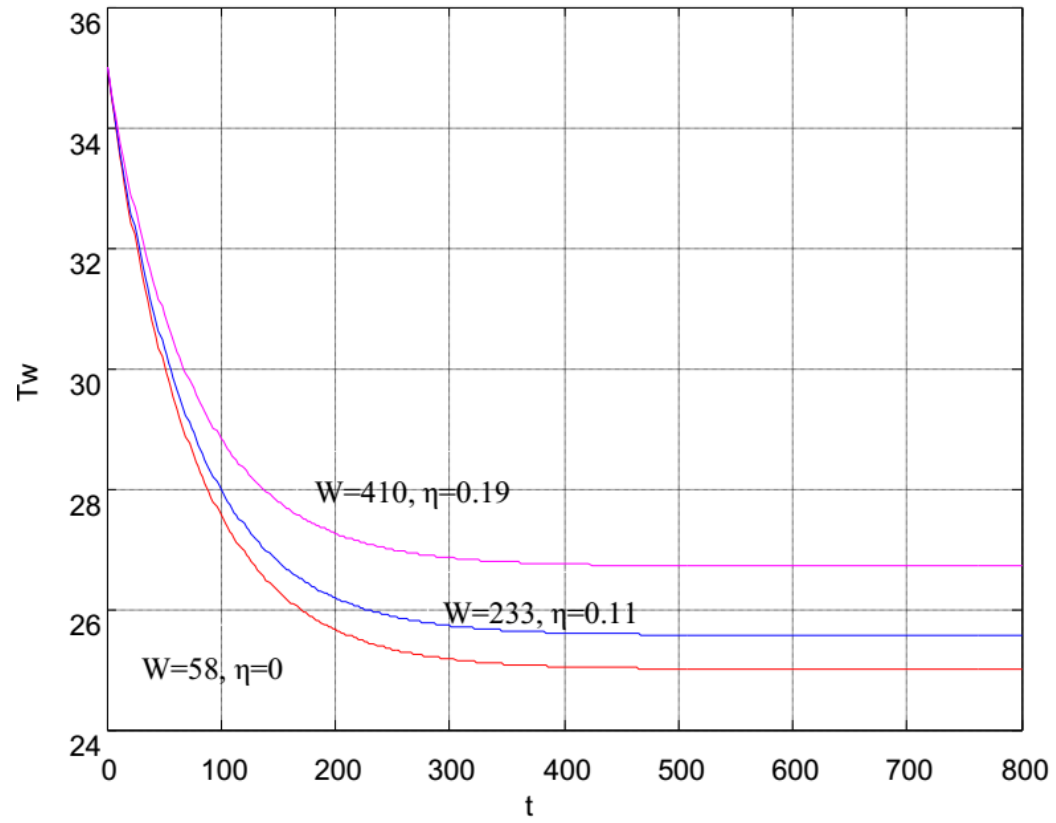

Fig. 3 Effect of the bather's movement on the temperature of the water

The heat dissipation factor B is related to the velocity of water flow and the size of the human body. The human body is regarded as an ellipse plane. Heat dissipation factor $B$ and their relationships are shown in the following Tables 1 [4].

Table 1 The relationship between the angle and the heat dissipation factor $B$

\begin{tabular}{cc}
\hline$\theta$ & $B$ \\
\hline 8.75 & 18.3 \\
34.29 & 17.2 \\
60 & 14.9 \\
85.71 & 11 \\
94.29 & 9.9 \\
120 & 7.6 \\
145.71 & 4.2 \\
171.43 & 1 \\
\hline
\end{tabular}

The relationship between the size of elliptic plane and the heat dissipation factor $B$ is shown as Table 2. 
Table 2 The relationship between the size of elliptic plane and the heat dissipation factor $B$

\begin{tabular}{lllc}
\multicolumn{3}{c}{ accordingly } \\
\hline$a$ & \multicolumn{2}{c}{$b$} & \multicolumn{2}{c}{$B$} \\
\hline & 0.1 & 0.1 & 15.32 \\
0.15 & 0.1 & 12.22 \\
0.2 & 0.15 & 10.17 \\
0.25 & 0.2 & 8.97 \\
0.3 & 0.25 & 8.2 \\
0.3 & 0.3 & 7.99 \\
\hline
\end{tabular}

The effect of heat dissipation factor $B$ on the temperature of the water is shown as Fig.4. The convective heat transfer between man and water is positively correlated with the heat dissipation factor $B$. Decreasing the $B$ can reduce the heat transfer between the human and the hot water. Reducing heat transfer and can lower the temperature drop rate. From the analysis, let people as much as possible to lie down, to achieve the purpose of saving water.

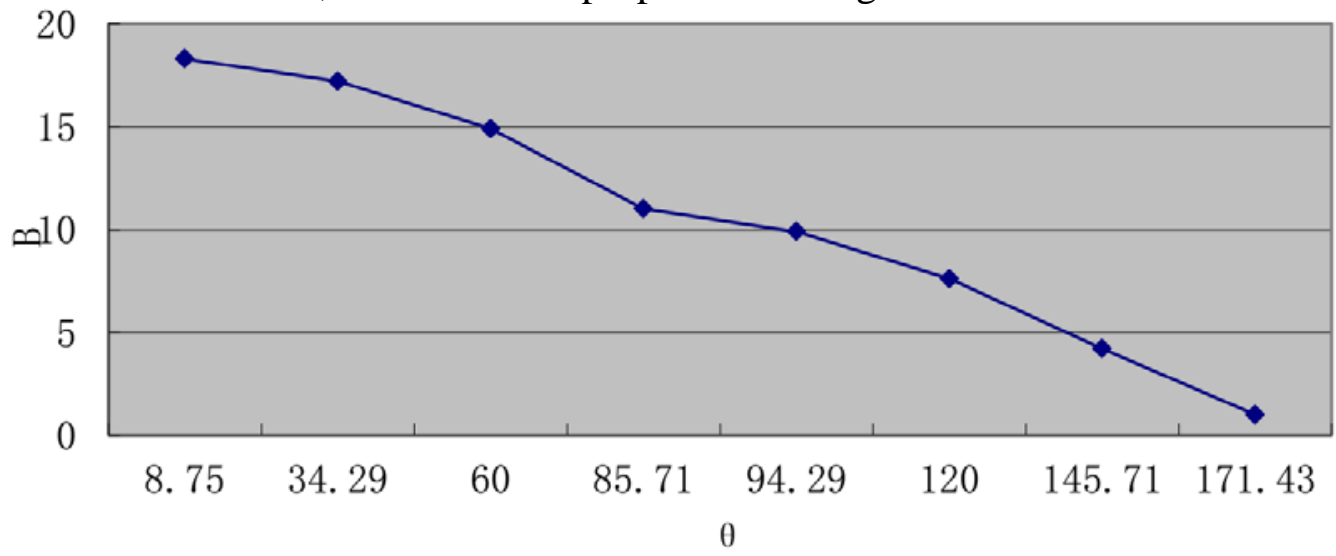

Fig. 4 The effect of heat dissipation factor $B$ on the temperature of the water

\section{Temperature Model of Bathtub Water when Water Flows into Bathtub}

We just turned the tap when water temperature drop. At the same time, the bath water is running over. So the relation between the temperature of the water in the bathtub and time changes to be [5].

$$
C_{P 1} M \frac{d T_{w}}{d t}=-\sigma \times \varepsilon_{w} \times\left(273.15+T_{w}\right)^{4} \times A-0.47 f_{w z} \times\left(T_{w}-T_{a}\right)-C_{p} q_{m}\left(T_{0}-T_{w}\right)-C_{p} q_{m} T_{w}
$$

Because the purpose of adding water is to keep the temperature constant. So you can use other variables to represent the flow rate.

$$
\frac{-\sigma \times \varepsilon_{w} \times\left(273.15+T_{w}\right)^{4} \times A-0.47 f_{w z} \times\left(T_{w}-T_{a}\right) \times A-\left(T_{w}-T_{a}\right)+\left(T_{w}-T_{R}\right) h_{c} S_{R}}{C_{p}\left(T_{s}-T_{w}\right)-C_{p} T_{w}}
$$

\section{The Shape of the Bathtub}

In order to make the surface area of the bathtub as small as possible, the following models can be established [6].

Assuming the volume $\mathrm{V}=2$ of the bathtub. Volume formula of rectangular bathtub:

$$
V=X \times Y \times h
$$

The formula for the surface area of the Cylinder bathtub:

$$
V=\pi \times r^{2} \times h
$$

Volume formula of Cylinder bathtub:

$$
\mathrm{S}=2 \times \pi \times r \times h+\pi \times r^{2}
$$

We take the actual size of the bathtub and the size of the human body into account, so we make some limits as follow. 
Rectangular bathtub is

$$
0.5 \ll h \ll 0.8(1 \ll x ; 2 \ll y)
$$

Cylinder bathtub is

$$
0.5 \ll h \ll 0.8(1 \ll r)
$$

\section{Conclusion}

By calculating, we can get that the smallest surface area is $7.66 \mathrm{~m}^{2}$ when Volume of rectangular bathtub is $2 \mathrm{~m}^{3}$. We can also get $x=1.414 \mathrm{~m}, y=2 \mathrm{~m}, h=0.707 \mathrm{~m}$.

Using the same methods, we can get that the smallest surface area is $7.14 \mathrm{~m}^{2}$ when volume of cylinder bathtub is $2 \mathrm{~m}^{3}$.we can also get $r=1 \mathrm{~m}, h=0.637 \mathrm{~m}$.

Therefore, in order to maintain the temperature of water in the bathtub, we propose to use a cylinder bathtub with a smaller surface area.

We also conclude some ways to reduce the total water.

- Increasing the thickness of the bathtub

- Reducing the surface area of the bathtub

- Increasing of human activities in the bathtub appropriately

- Bather should use the supine position as far as possible.

- Using cylinder bathtub

\section{References}

[1] Bednarz T P, Lei C, Patterson J C. An experimental study of unsteady natural convection in a reservoir model cooled from the water surface [J]. Experimental Thermal \& Fluid Science, 2008, 32(3):844-856.

[2] Sposito G, White R E, Darrah P R, et al. A Transfer Function Model of Solute Transport Through Soil: 3. The Convection-Dispersion Equation [J]. Water Resources Research, 1986, 22(22):255-262.

[3] Patankar S V. Numerical heat transfer and fluid flow [M]// false. 1980:125-126.

[4] Sharma A, Tyagi V V, Chen C R, et al. Review on thermal energy storage with phase change materials and applications[J]. Renewable \& Sustainable Energy Reviews, 2009, 13(2):318-345.

[5] Kenisarin M M. High-temperature phase change materials for thermal energy storage [J]. Renewable \& Sustainable Energy Reviews, 2010, 14(3):955-970.

[6] Cárdenas B, León N. High temperature latent heat thermal energy storage: Phase change materials, design considerations and performance enhancement techniques [J]. Renewable \& Sustainable Energy Reviews, 2013, 27(6):724-737. 